\begin{tabular}{||ll||}
\hline Citation/Reference & $\begin{array}{l}\text { Carolina Varon, Jesus Lazaro, Juan Bolea, Alberto Hernando, Jordi Aguilo, } \\
\text { EduardoGil, Sabine Van Huffel, Raquel Bailon (2018), } \\
\text { Unconstrained Estimation of HRV Indices after Removing } \\
\text { Respiratory Influences from Heart Rate } \\
\text { IEEE Journal of Biomedical and Health Informatics. Accepted }\end{array}$ \\
\hline Archived version & $\begin{array}{l}\text { Author manuscript: the content is identical to the content of the published } \\
\text { paper, but without the final typesetting by the publisher }\end{array}$ \\
\hline Published version & Not yet available \\
\hline Journal homepage & $\underline{\text { https://jbhi.embs.org } /}$ \\
\hline Author contact & your phone number $+32(0) 16326417$ \\
\hline IR & $\underline{\text { Not yet available }}$ \\
\hline
\end{tabular}




\title{
Unconstrained Estimation of HRV Indices after Removing Respiratory Influences from Heart Rate
}

\author{
Carolina Varon, Member, IEEE, Jesús Lázaro, Member, IEEE, Juan Bolea, Alberto Hernando, Member, IEEE, \\ Jordi Aguiló, Eduardo Gil, Sabine Van Huffel, Fellow, IEEE, and Raquel Bailón
}

\begin{abstract}
Objective: This paper proposes an approach to better estimate the sympathovagal balance (SB) and the respiratory sinus arrhythmia (RSA) after separating respiratory influences from the heart rate (HR). Methods: The separation is performed using orthogonal subspace projections and the approach is first tested using simulated HR and respiratory signals with different spectral properties. Then, RSA and SB are estimated during autonomic blockade and stress using the proposed approach and the classical heart rate variability (HRV) analysis. Both real and ECG-derived respiration (EDR) are used and the reliability of the EDR is evaluated. Results: Mean absolute percentage errors lower than $1 \%$ were obtained after removing previously known respiratory signals from simulated HR. The proposed indices were able to improve the quantification of SB during autonomic withdrawal. In the stress data, differences $(p<0.003)$ among relaxed and stressful phases were found with the proposed approach, using both the real respiration and the EDR, but they disappeared when using the classical HRV. Conclusion: A better assessment of the autonomic nervous system' response to pharmacological blockade and stress can be achieved after removing respiratory influences from $H R$, and this can be done using either the real respiration or the EDR. Significance: This work can be used to better identify vagal withdrawal and increased sympathetic activation when the classical HRV analysis fails due to the respiratory influences on HR. Furthermore, it can be computed using only the ECG, which is an advantage when developing wearable systems with limited number of sensors.
\end{abstract}

Index Terms-Respiratory Sinus Arrhythmia, Sympathovagal Balance, Stress.

Manuscript received April 19, 2005; revised August 26, 2015.

This work is supported by: OSA+. iMinds Medical Information Technologies: ICON HBC.2016.0167. European Research Council: The research leading to these results has received funding from the European Research Council under the European Union's Seventh Framework Programme (FP7/20072013) / ERC Advanced Grant: BIOTENSORS (n³39804). European Union's Framework Programme for Research and Innovation Horizon 2020 (20142020) under the Marie Skłodowska-Curie Grant Agreement No. 745755 UZ2018-TEC-05 (University of Zaragoza), T96 (Government of Aragon and European Social Fund), CIBER-BBN (Instituto de Salud Carlos III and FEDER). This paper reflects only the authors' views and the Union is not liable for any use that may be made of the contained information. Carolina Varon is a postdoctoral fellow of the Research Foundation-Flanders (FWO).

C. Varon and S. Van Huffel are with the Department of Electrical Engineering-ESAT, STADIUS Center for Dynamical Systems, Signal Processing and Data Analytics, KU Leuven, and imec, B-3001 Leuven, Belgium, (e-mail: carolina.varon, sabine.vanhuffel @esat.kuleuven.be).

J. Lázaro is with the Department of Electrical Engineering of University of Connecticut, Storrs CT, USA, (e-mail: jlazarop@unizar.es).

A. Hernando is with the Centro Universitario de la Defensa, Zaragoza, Spain, (e-mail: alb_her_1991@hotmail.com).

J. Aguiló is with the Microelectronics and Electronic Systems Department, Autonomous University of Barcelona, Bellaterra, Spain, (e-mail: jordi.aguilo@uab.es).

J. Lázaro, J. Bolea, E. Gil and R. Bailón are with the BSICoS Group, Aragón Institute of Engineering Research (I3A), IISAragon, University of Zaragoza and CIBER-BBN, Zaragoza, Spain, (e-mail: jlazarop, jbolea, edugilh, rbailon@unizar.es).

\section{INTRODUCTION}

$\mathbf{I}$ $\mathrm{T}$ is widely accepted that the classical heart rate variability (HRV) analysis allows to quantify the vagal and sympathetic modulations of the autonomic nervous system (ANS) [1]. This quantification can be done using the power spectrum of the HRV, where the power in the low frequency (LF) band defined between $0.04 \mathrm{~Hz}$ and $0.15 \mathrm{~Hz}$ is believed to quantify both sympathetic and parasympathetic activity. The high frequency (HF) band, on the other hand, defined in the range between $0.15 \mathrm{~Hz}$ and $0.4 \mathrm{~Hz}$, is assumed to purely describe vagal activity. The latter has been shown to quantify the modulations of heart rate driven by the respiration, or the so-called respiratory sinus arrhythmia (RSA) [2]. Even though this division of the power spectrum is well-standardized, it is restricted to cases when the respiratory rate falls within the HF band. Instead, if the respiratory rate is higher than the upper limit of the HF band, an underestimation of the vagal control can be achieved. This can be overcome after centering the HF band at the respiratory frequency, as done in [3]. Conversely, when the respiratory rate falls below the upper limit of the LF, the interpretation of the frequency parameters as sympathetic and/or parasympathetic activity is no longer possible [3]-[5]. In fact, if the respiratory frequency is within the LF band, the sympathetic activity is overestimated and the parasympathetic activity underestimated.

Typical approaches to quantify RSA are based on computing the spectral power around the respiratory frequency, or on RR interval values at inspiratory and expiratory phases [6], [7]. However, the estimation of respiratory frequency and of inspiratory and expiratory phases in highly nonstationary and broad-band respiration (e.g. while speaking) challenge the assessment of both sympathetic and vagal activities [8].

A consequence of the aforementioned limitations is the misinterpretation of the ratio $\mathrm{LF} / \mathrm{HF}$, which has been proposed as an index of sympathovagal balance (SB) [9]. Even though the quantification of the modulation of the ANS using a single number is challenging and often controversial [10], [11], the SB has been widely used in literature despite its limited interpretability. For instance, it has been shown that during tilt test, stress, exercise, apnea, and epilepsy, an increased SB can be identified using this ratio [12]-[16].

Taking into account these limitations of the classical HRV analysis, it is clear that an approach to better estimate the RSA and the SB under different respiratory patterns must be developed [4], [17]-[20]. In this context, this study proposes to use the heart rate decomposition based on orthogonal subspace 
projections (OSP) formulated in [21], [22] and adapted for cardiorespiratory analysis in [4], [18]. This methodology separates the respiratory influences from the heart rate, providing a novel approach to better estimate the RSA and the SB. Moreover, this approach will be first tested in a simulated dataset in order to verify that the heart rate decomposition is able to separate respiratory influences at different frequencies and with different spectral characteristics. Then, the approach will be tested on a pharmacological dataset where autonomic blockade was induced [23], and on the stress dataset used in [3]. These datasets will be used in order to evaluate if the improved quantification can indeed identify the increased sympathetic activation and vagal withdrawal expected during autonomic blockade (i.e. using atropine) and stress, even during phases where the subjects were speaking.

The proposed estimation of the RSA and the SB will also be performed using the ECG-derived respiration (EDR). Here, a state-of-the-art algorithm will be used to evaluate whether the approach could be used when only the ECG is available. As a result, the applicability of the proposed approach on wearable systems, which could be developed for the assessment and management of stress, will be identified.

The remainder of this paper is organized as follows. Section II describes the heart rate decomposition, the simulation studies, the real datasets, and the EDR algorithm. In Section II-B, the novel approach to calculate the RSA and the SB is proposed. Section III describes the results, which are discussed in Section IV. Conclusions are presented in Section V.

\section{Methodology}

\section{A. Heart Rate Decomposition}

The HRV signal can be decomposed into two different components, one respiratory component, describing all variations related to respiration, and one residual component, describing all dynamics modulated by other mechanisms different from respiration. In fact, the residual component describes dynamics modulated by the sympathetic nervous system, and other (possible) vagal modulators unrelated to respiration. The heart rate decomposition can be achieved by means of OSP as in [4], [18], and its formulation is described as follows.

Given are two physiological signals $\mathbf{X}=\left\{x_{i}\right\}_{i=1}^{N}$ and $\mathbf{Y}=$ $\left\{y_{i}\right\}_{i=1}^{N}$, where $\mathbf{X}$ corresponds to the respiratory signal, $\mathbf{Y}$ corresponds to the HRV signal, and $N$ is the length of the signals. In this study, it is assumed that the respiratory signal drives changes in the HRV signal, which is then seen as the target signal. In order to extract all dynamics of the heart rate that are linearly related to respiration, $\mathbf{Y}$ can be projected onto a subspace $\mathbb{V}$ defined by all variations in $\mathbf{X}$. This subspace is constructed using the respiratory signal $\mathbf{X}$ and its delayed versions, going from 1 to $m$ samples [18]. In other words, a matrix $\mathbf{V}$ spanning the subspace $\mathbb{V}$ is constructed as $\mathbf{V}=$ $\left[\mathbf{X}_{0}, \mathbf{X}_{1}, \ldots, \mathbf{X}_{m}\right]$, with $\mathbf{X}_{d}=\left[x_{d+1}, x_{d+2}, \ldots, x_{N-m+d}\right]^{T}$ and $d=0, \ldots, m$. In this study, the value of $m$ (i.e., model order) is defined as the minimum amount of delays obtained using both the minimum description length (MDL) principle and the Akaike Information Criterion (AIC), with a maximum delay of $10 \mathrm{~s}$, which corresponds to a respiratory frequency of $0.1 \mathrm{~Hz}$. Nevertheless, it is important to keep in mind that respiratory information could be contained below this limit, therefore, the maximum model order should be defined for each case, based on this information.

After creating the matrix $\mathbf{V}$, the $\mathrm{HRV}$ signal $\mathbf{Y}$ can be projected onto the respiratory subspace $\mathbb{V}$ by means of

$$
\mathbf{Y}_{\mathbf{X}}=\mathbf{P Y}
$$

with $\mathbf{P}$ a projection matrix defined as

$$
\mathbf{P}=\mathbf{V}\left(\mathbf{V}^{T} \mathbf{V}\right)^{-1} \mathbf{V}^{T}
$$

As a result, all dynamics of $\mathbf{Y}$ linearly related to $\mathbf{X}$ are described in the respiratory component denoted by $\mathbf{Y}_{\mathbf{X}}$. Furthermore, an orthogonal component $\mathbf{Y}_{\perp}$, which is related to other heart rate modulators can be computed as $\mathbf{Y}_{\perp}=\mathbf{Y}-\mathbf{Y}_{\mathbf{X}}$.

This heart rate decomposition can be seen as a generalization of the cardiorespiratory model proposed in [17], which is based on an autoregressive moving average model with exogenous inputs (ARMAX) and solved using least-squares. The full heart rate decomposition is summarized in Algorithm 1.

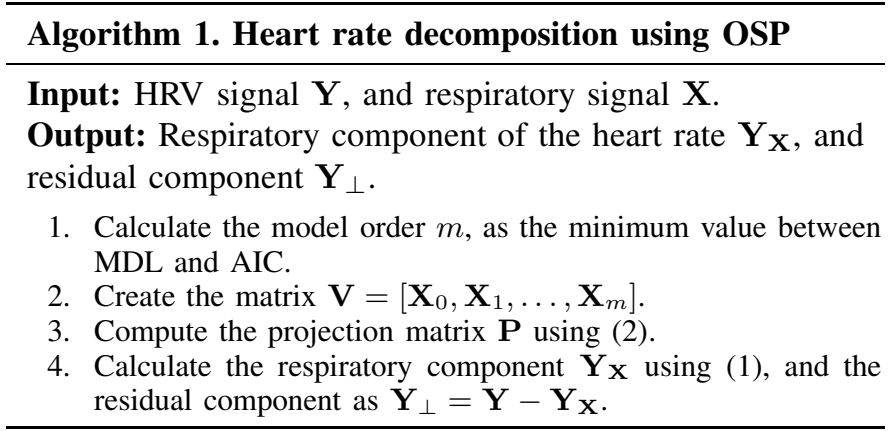

The two different components of the heart rate can be characterized using the classical frequency domain HRV features. At this point, the LF is quantified from $0.04 \mathrm{~Hz}$ to $0.15 \mathrm{~Hz}$, and the HF can be either defined from $0.15 \mathrm{~Hz}$ to $0.4 \mathrm{~Hz}$, or using the extended band from $0.15 \mathrm{~Hz}$ to half the mean heart rate as in [5]. In addition, the relative power of each component with respect to the original HRV signal can be calculated as

$$
\mathcal{P}_{\mathbf{X}}=\frac{\mathbf{Y}_{\mathbf{X}}^{\prime} \mathbf{Y}_{\mathbf{X}}}{\mathbf{Y}^{\prime} \mathbf{Y}} \quad \text { and } \quad \mathcal{P}_{\perp}=\frac{\mathbf{Y}_{\perp}^{\prime} \mathbf{Y}_{\perp}}{\mathbf{Y}^{\prime} \mathbf{Y}}
$$

These relative powers can be used to get an indication of how much information is shared between respiration and heart rate. For instance, when $\mathcal{P}_{\mathbf{X}}>\mathcal{P}_{\perp}$, most of the variations in the heart rate can be described by changes in the respiration.

\section{B. Unconstrained estimation of the RSA and HRV indices}

The main result of the heart rate decomposition described before is the separation of the linear respiratory influences from the heart rate. As a result, a better quantification of the LF power of HRV unrelated to respiration can be achieved, which on its turn can improve the assessment of both the RSA and the SB as recognized in [1]. In fact, the relative power of the respiratory component of the $\mathrm{HR}$, namely, $\mathcal{P}_{\mathbf{X}}$, gives an indication of strength of cardiorespiratory interactions, while the total power of the respiratory component $\mathbf{Y}_{\mathbf{X}}$, can be 
used as an unconstrained index for RSA assessment. This index is unconstrained because no assumption is made on the respiratory frequency nor on the morphology of the respiration, which are assumptions often made when quantifying the RSA. For instance, all the methods evaluated in [7] require either the definition of the inspiratory and expiratory phases or that the respiratory rate falls within a defined frequency band. The latter is also assumed in methods based on transfer function [6].

Since the respiratory influences are removed from the heart rate, it is assumed that the residual heart rate component, namely $\mathbf{Y}_{\perp}$, contains information (linearly) unrelated to respiration. This means that the LF band of this component might quantify the sympathetic activity in a better way. Therefore, a better assessment of the ANS can be achieved.

It is well-known that the SB is defined as the ratio between the power of LF modulators of HRV and the component of the HRV. The LF modulators are mainly associated with the sympathetic activation of the ANS, while the HF component mainly represents respiratory modulation [9]. To guarantee that this index indeed quantifies the ratio between sympathetic modulation and the respiratory modulation, this study proposes to compute an unconstrained version of the SB as

$$
\mathrm{SB}_{\mathrm{u}}=\frac{\mathrm{LF}_{\perp}}{\mathrm{LF}_{\mathbf{X}}+\mathrm{HF}_{\mathbf{X}}}
$$

with $\mathrm{LF}_{\mathbf{X}}$ and $\mathrm{HF}_{\mathbf{X}}$ estimated from the PSD of $\mathbf{Y}_{\mathbf{X}}$. As a result, all linear respiratory influences are removed from the quantification of the sympathetic activation (i.e. numerator).

In order to quantify the added value of $\mathrm{SB}_{\mathrm{u}}$ with respect to the classical calculation of the sympathovagal balance, namely $\mathrm{SB}=\mathrm{LF} / \mathrm{HF}$ (where LF and HF are computed from the PSD of $\mathrm{Y})$, both indices will be computed for different stress phases and during autonomic blockade. The datasets used for this purpose will be described in Section II-D.

\section{Simulation Studies}

The heart rate decomposition used in this study is based on the assumption that all respiratory information is described in the respiratory subspace $\mathbb{V}$. In order to determine if this is indeed the case, two different simulations are designed, where an artificial HRV signal $\mathbf{Y}$ is decomposed using respiratory signals with different properties.

The signal $\mathbf{Y}$ is generated as indicated in Figure 1. First, two random signals are generated as $\mathbf{Z}_{1}=\left\{z_{1 i}\right\}_{i=1}^{m}$ and $\mathbf{Z}_{2}=$ $\left\{z_{2 i}\right\}_{i=1}^{m}$, with $m$ the length of the signals, $\mathbf{Z}_{1} \sim N(0,1)$, and $\mathbf{Z}_{2} \sim N(0,1)$. Five minutes segments are generated with a sampling frequency of $5 \mathrm{~Hz}$. Then, $\mathbf{Z}_{1}$ and $\mathbf{Z}_{2}$ are band-pass filtered using the limits of the LF band and the LF+HF bands, respectively. This is done to simulate the sympathetic modulations in $\mathbf{Y}_{s}$, and the possible parasympathetic modulations unrelated to respiration in $\mathbf{Y}_{p}$. Although the latter might be expected to disappear when no respiratory influence (i.e. RSA) is present, it is still a matter of debate whether the vagal tone, quantified by the HF band of HRV, is exclusively related to changes in respiration [24]. For this reason, $\mathbf{Y}_{p}$ still contains information at the HF band.

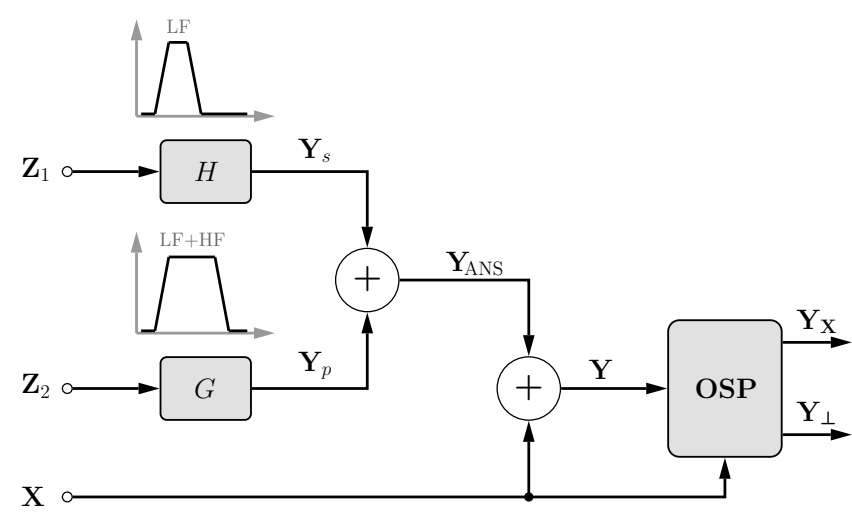

Fig. 1. Procedure to generate the simulated data and to perform the heart rate decomposition using OSP.

At this point, $\mathbf{Y}_{s}$ and $\mathbf{Y}_{p}$ are added to obtain the autonomic nervous activity excluding any respiratory component, as $\mathbf{Y}_{\mathrm{ANS}}=\mathbf{Y}_{s}+\mathbf{Y}_{p}$. Note that $\mathbf{Y}_{\mathrm{ANS}}$ only represents ANS activity which is assumed to be unrelated to respiration, hence, it does not describe possible respiratory modulations of the sympathetic outflow, as reported in [25]. Moreover, since the two ANS components are known to work in tandem, the ratio between the LF and HF powers is modified between 0.8 and 5 to simulate either vagal or sympathetic dominance, respectively.

Once the ANS signal unrelated to respiration is simulated, all respiratory influences, described by $\mathbf{X}$, are added to $\mathbf{Y}_{\text {ANs }}$ in order to obtain the HRV signal $\mathbf{Y}$. Both $\mathbf{Y}_{\mathrm{ANs}}$ and $\mathbf{X}$ are normalized to zero mean and unit variance before they are added to generate $\mathbf{Y}$. Furthermore, two different types of respiratory patterns are considered and they will be described next.

1) Monocomponent Respiratory Signal: For the first simulation, the respiratory signal is defined as $\mathbf{X}=\left\{x_{i}\right\}_{i=1}^{N}$ with $x_{i}=A \sin \left(2 \pi f \frac{i}{f_{s}}\right)+e_{i}, f_{s}$ the sampling frequency, $0.1 \mathrm{~Hz} \leq f \leq 0.4 \mathrm{~Hz}$, and the error $\mathbf{E}=\left\{e_{i}\right\}_{i=1}^{N}$ with $\mathbf{E} \sim \mathcal{N}(0,1)$. The respiratory frequency $f$ remains constant for each realization, see Figure 2(a). Figure 2(b) depicts the power spectral density (PSD) of the simulated HRV and respiratory signals. The PSDs are calculated using the Welch's algorithm with a Hamming window of $60 \mathrm{~s}$, an overlap of 40 s, and 1024 points. Note that in the LF band, both $\mathbf{Y}_{\text {ANS }}$ and $\mathbf{X}$ have a peak at $0.1 \mathrm{~Hz}$. Therefore, the main question here is whether the decomposition approach is able to separate the respiratory influences from the pure sympathetic modulations, contained in $\mathbf{Y}_{s}$, when they completely overlap. If this is the case, then a better assessment of the ANS can be achieved.

In order to evaluate the performance of the decomposition throughout the whole frequency range of interest, namely LF and HF, $\mathbf{Y}_{\mathrm{ANS}}$ will be linearly influenced by different respiratory frequencies that will range between $0.1 \mathrm{~Hz}$ and $0.4 \mathrm{~Hz}$. This will be done 31 times for 1000 different $\mathbf{Y}_{\text {ans }}$ signals. The reason to use 31 values is to obtain frequencies from $0.1 \mathrm{~Hz}$ to $0.4 \mathrm{~Hz}$ in steps of $0.01 \mathrm{~Hz}$. The step size was selected arbitrarily in order to evaluate an integer number of frequency values within the band. 


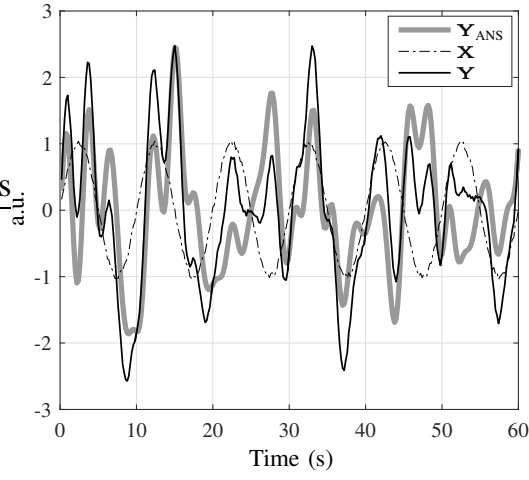

(a)

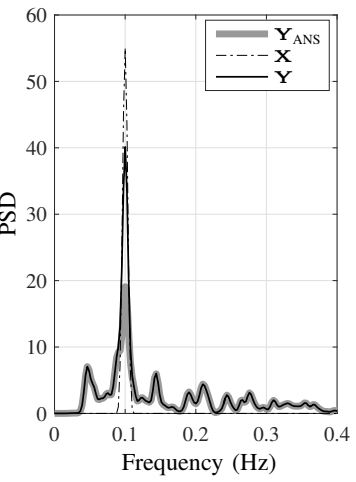

(b)

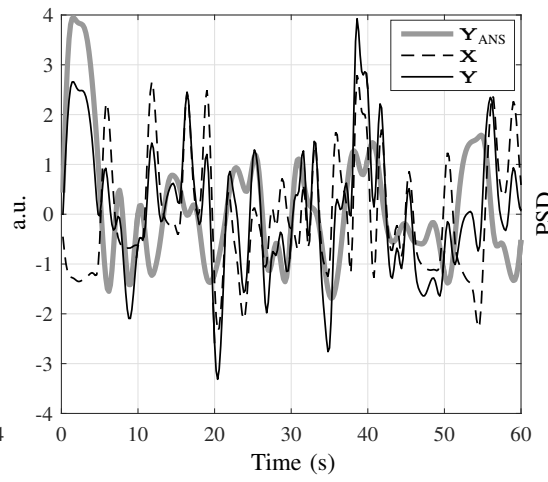

(c)

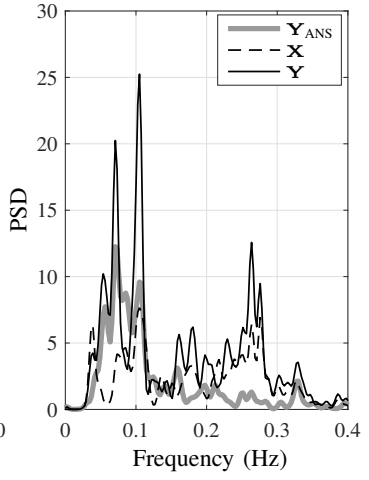

(d)

Fig. 2. Example of the simulated signals and their corresponding PSDs. (a) simulated signals with the first approach, where both the respiratory signal $\mathbf{X}$ and the ANS signal $\mathbf{Y}_{\mathrm{ANS}}$ have components at $0.1 \mathrm{~Hz}$. Hence, the spectrum in (b) of the respiration completely overlaps with the simulated sympathetic activation in the HRV signal Y, simulating a strong linear coupling. (c) Simulated signals using the second approach, and their corresponding PSDs (d). The respiratory signal $\mathbf{X}$ has a broadband spectrum that overlaps with the one of $\mathbf{Y}_{\text {ANs }}$.

2) Broadband Respiration: The second simulation consists of influencing the ANS signal, $\mathbf{Y}_{\mathrm{ANS}}$, with real respiratory signals with a broader frequency spectrum. The respiratory signals of the Fantasia dataset [26], publicly available in Physionet, will be used as $\mathbf{X}$ and they will be added to $\mathbf{Y}_{\text {ANs }}$. This dataset includes respiratory effort that was recorded using a respiratory belt around the thorax of 40 volunteers while watching the movie Fantasia (Disney, 1940). The signals were recorded for about 120 minutes with a sampling frequency of $250 \mathrm{~Hz}$. For the simulation study, all signals were first bandpass-filtered using cutoff frequencies of $0.03 \mathrm{~Hz}$ and 0.9 Hz. After that, they were downsampled at $5 \mathrm{~Hz}$ and segmented into epochs of 5 minutes. In total, 932 segments were extracted, which means that the simulation was performed 932 times with 1000 different $\mathbf{Y}_{\text {ANs }}$ signals each time. An example $\mathbf{Y}_{\mathrm{ANS}}$ signal is shown in Figure 2(c) together with one segment extracted from the Fantasia dataset. Both the signals in time and the PSDs (see Figure 2(d)) are shown. At this point, the question is whether the ANS signal $\mathbf{Y}_{\mathrm{ANS}}$ can be reconstructed when subjects are breathing naturally, and when the spectrum of the respiration is broader as it occurs while the subject speaks. In these cases it is not possible to assess the RSA using the traditional algorithms since neither the respiratory frequency nor the inspiratory/expiratory phases can be determined.

The goal of the aforementioned simulations is to determine if the algorithm is capable of reconstructing the ANS signal $\mathbf{Y}_{\text {ANS }}$ after removing all (possibly overlapping) respiratory influences $(\mathbf{X})$ from the heart rate $(\mathbf{Y})$. Each signal $\mathbf{Y}$ will be decomposed into $\mathbf{Y}_{\mathbf{X}}$ and $\mathbf{Y}_{\perp}$ and the performance of the algorithm will be measured in three ways. First, the mean absolute percentage error (MAPE) between $\mathbf{Y}_{\perp}$ and $\mathbf{Y}_{\mathrm{ANS}}$ will be calculated. Second, the LF and HF powers of both the $\mathbf{Y}_{\perp}$ and the $\mathbf{Y}_{\mathrm{ANS}}$ signals will be compared. In order to do so, the percentage errors defined as

$$
\begin{aligned}
& e_{\mathrm{LF}}=\frac{\left|\mathrm{LF}_{\mathrm{ANS}}-\mathrm{LF}_{\perp}\right|}{\mathrm{LF}_{\mathrm{ANS}}} \times 100 \text { and } \\
& e_{\mathrm{HF}}=\frac{\left|\mathrm{HF}_{\mathrm{ANS}}-\mathrm{HF}_{\perp}\right|}{\mathrm{HF}_{\mathrm{ANS}}} \times 100
\end{aligned}
$$

will be computed for each decomposition, with $\mathrm{LF}_{\mathrm{ANS}}$ and $\mathrm{HF}_{\mathrm{ANS}}$ the frequency parameters of $\mathbf{Y}_{\mathrm{ANS}}$, and $\mathrm{LF}_{\perp}$ and $\mathrm{HF}_{\perp}$ the frequency parameters of $\mathbf{Y}_{\perp}$. Finally, the error in the normalized LF power will be computed as

$$
e_{n}=\frac{\left|\mathrm{LFn}_{\mathrm{ANS}}-\mathrm{LFn}_{\perp}\right|}{\mathrm{LFn}_{\mathrm{ANS}}} \times 100
$$

with

$$
\mathrm{LFn}_{\mathrm{ANS}}=\frac{\mathrm{LF}_{\mathrm{ANS}}}{\mathrm{LF}_{\mathrm{ANS}}+\mathrm{HF}_{\mathrm{ANS}}} \text { and } \mathrm{LFn}_{\perp}=\frac{\mathrm{LF}_{\perp}}{\mathrm{LF}_{\perp}+\mathrm{HF}_{\perp}} \text {. }
$$

The values of LF and HF for all signals will be computed after integrating their PSDs in the corresponding bands, namely, $0.04-0.15 \mathrm{~Hz}$ for $\mathrm{LF}$ and $0.15-0.4 \mathrm{~Hz}$ for HF. At this point, the upper limit of the $\mathrm{HF}$ band is set to $0.4 \mathrm{~Hz}$ since $\mathbf{Y}_{\mathrm{ANS}}$ is simulated in such way that it does not contain information above that frequency. All PSDs will be calculated using the Welch's algorithm with a Hamming window of $60 \mathrm{~s}$, an overlap of $40 \mathrm{~s}$, and 1024 points.

\section{Real datasets}

Two datasets will be used to test the quantification of the RSA and sympathovagal balance after decomposing the heart rate.

1) Pharmacological autonomic blockade dataset: This dataset was recorded at the Massachusetts Institute of Technology and the experimental protocol is described in detail in [23] and it includes single pharmacological blockade of the sympathetic and parasympathetic branches of the ANS. The dataset consists of ECG and respiratory effort signals sampled at $360 \mathrm{~Hz}$ and recorded from 13 male volunteers (ages 19-38 years, $21 \pm 4.4$ years) with no history of cardiopulmonary disease. Subjects were first in a supine position and both signals were recorded during 7 minutes. Then, subjects were moved to a standing position, and after 5 minutes of adaptation the signals were recorded for 7 more minutes. These two phases will be called supine control (SUC) and standing control (STC) since no drug was administered. After these control phases, subjects were divided into two groups. One group of 7 subjects received atropine $(0.03 \mathrm{mg} / \mathrm{kg})$ for complete 
parasympathetic blockade, and the other group of 6 subjects received propranolol $(0.2 \mathrm{mg} / \mathrm{kg})$ for complete reduction of sympathetic activity. After 10 minutes for equilibration, the protocol of supine and standing positions described above was repeated. These new phases will be called SUA and STA or SUP and STP, where A and P refer to atropine and propranolol, respectively. In total, 4 segments of 7 minutes were analyzed per subject: 2 control segments and 2 with single blockade. During these phases, subjects were asked to initiate a breathing cycle after hearing a tone. These tones were irregular with a minimum and maximum intervals of 1 and $15 \mathrm{~s}$, respectively. Despite this imposed respiratory pattern, subjects were allowed to vary the depth and shape of each breath in order to guarantee normal ventilation. With this in mind, the maximum model order for the heart rate decomposition will be $15 \mathrm{~s}$. Furthermore, since the breathing protocol was imposed in such a way that the power spectrum of the respiratory drive was nearly flat [23], the model order was selected as the maximum value between AIC and MDL, instead of the minimum. As a result, a more complex model can be built to better characterize the random effect in the respiratory signals present in many phases.

This dataset will allow to test the proposed quantification of RSA and SB when there is complete parasympathetic withdrawal during STA (i.e. pure sympathetic modulation), and when there is pure parasympathetic modulation during SUP.

2) Stress dataset: This dataset was collected at the University of Zaragoza (UZ) and the Autonomous University of Barcelona (UAB) and was approved by both Ethics Committees. The dataset consists of ECG and respiratory effort recorded from 46 volunteers. The mean age of the volunteers was $21.76 \pm 4.48$ years (range 18-32 years), and 18 were men and 28 female. The ECG was sampled at $1 \mathrm{kHz}$ and the respiration at $250 \mathrm{~Hz}$. All volunteers underwent a stress session, where emotional stress was induced by means of a modified Trier Social Stress Test [3]. The tests include the following phases:

- Baseline (BL). For about 10 minutes, the subject listens to a relaxing audio

- Story telling (ST). 3 stories are told to the subject and he/she is asked to remember as many details as possible.

- Memory task (MT). The subject is asked to tell in front of a camera, all details that he/she remembered from the ST phase.

- Stress anticipation (SA). The subject is asked to wait for about 10 minutes for the results of the evaluation of the MT phase.

- Video exposition (VE). The video recorded during the MT phase is shown to the subject together with another video, where an actor repeats the stories in a perfect way. The idea is to make a comparison between the "poor" performance of the subject and a much better performance of the actor.

- Arithmetic task (AT): The subject is asked to count down out loud from 1022 in steps of 13 . Whenever the subject made a mistake, he/she was asked to start again from 1022. A constraint of 5 minutes was imposed in order to induce stress in the subject. No subject succeeded to finalize the countdown.

Of the 46 volunteers, 11 were excluded from the study because some of their phases were corrupted by technical artefacts. Therefore, 35 subjects were included in the study, and the average duration of the phases is $11.53 \pm 1.4$ minutes for $\mathrm{BL}, 1.68 \pm 0.5$ minutes for ST, $1.96 \pm 0.18$ minutes for MT, $11.18 \pm 1.2$ minutes for SA, $3.20 \pm 0.48$ minutes for $\mathrm{VE}$, and $5.49 \pm 0.5$ minutes for AT.

Stress induction was confirmed by means of multiple psychometric scores, such as the Perceived Stress Scale, Visual Analogue Scale, and the State-Trait Anxiety Inventory [3].

\section{E. Application on real data}

In this application, the relative powers described in (3) and the HRV parameters, including the sympathovagal balance, for each heart rate component will be computed for the different phases of the two datasets described before. This will be done using the real respiratory effort and the EDR signal.

1) Pre-processing: The respiratory signals were first bandpass filtered using a Butterworth filter with cutoff frequencies of $0.03 \mathrm{~Hz}$ and $0.9 \mathrm{~Hz}$. Then, they were downsampled at $4 \mathrm{~Hz}$, and normalized with zero mean and unit variance. The ECG signals, on the other hand, were used to find the location of the R-peaks by means of the algorithm described in [18]. After that, ectopic, missed and false peaks were corrected by means of the integral pulse frequency modulation (IPFM) model [27]. Then, the time series were corrected for variations in the mean heart rate, and the HRV signals were computed as described in [3]. Finally, the HRV signals were resampled at $4 \mathrm{~Hz}$ and band-pass filtered as it was done for the respiratory signals.

For the stress dataset, the transient behavior between phases was removed by using only the middle segments of each phase. In other words, the first and last seconds of each phase were removed, namely, 1 minute for $\mathrm{BL}$ and SA, 5 seconds for ST and MT, and 10 seconds for VE and AT. For the pharmacological dataset all phases were recorded after minutes of equilibration, hence, all 7 minutes of recordings per phase were used for the analysis.

2) Frequency parameters: Each phase was decomposed using the proposed approach, and each component was characterized by the relative powers defined in (3), where the RSA was estimated using the total power in $\mathbf{Y}_{\mathbf{X}}$ in the extended $\mathrm{HF}$ band (from $0.15 \mathrm{~Hz}$ to half the mean heart rate). Furthermore, the powers of each component in the classical LF $(0.04-0.15$ $\mathrm{Hz}$ ) and in the extended $\mathrm{HF}$ band were computed together with their normalized versions. These values were then used to calculate the sympathovagal balance using both the classical and the unconstrained approach.

3) The ECG-Derived Respiration: The heart rate decomposition used in this study requires the definition of the respiratory subspace $\mathbb{V}$, which is constructed using the respiratory signal. Often, the recording of the respiration is associated with invasive and intrusive sensors such as belts, thermocouples, and thermistors. Although these sensors are regularly used in multiple medical tests, they are very much avoided in ambulatory settings. Reasons for this include the discomfort 
and the high costs associated with their use. With this in mind, different authors have shown that an approximated respiratory signal can be derived from the ECG in order to reduce the amount of sensors needed to assess the cardiorespiratory system [28]-[31]. This approximated signal, the so-called ECGderived respiration (EDR), is the result of the morphology changes of the ECG caused by the mechanical influence of the respiration. In other words, the movement of the chest during each breathing cycle changes the relative position of the electrodes with respect to the cardiac vector. On the other hand, the constant filling and emptying of the lungs causes variations in the electrical impedance of the chest. Both effects result in amplitude modulations of the ECG that are strongly associated with the breathing cycle.

In this study, the EDR algorithm based on kernel principal component analysis (kPCA) proposed in [30] will be used. kPCA allows to extract linear and nonlinear influences of respiration on the morphology of the ECG, which has been shown to be an advantage in the reconstruction of the respiratory signal from the ECG [30]. This algorithm takes into account variations in amplitudes of all the points of the QRS complexes, by first segmenting the QRSs using a symmetric window of $120 \mathrm{~ms}$ around the R-peaks. Then, all QRSs are organized in a matrix, and the mean variation of all the points is then calculated using kPCA. This is done by mapping the QRS matrix into a higher dimensional space by means of a kernel function. After that, the classical PCA is performed on the transformed dataset, and the first principal component is mapped back to the input space and then taken as the EDR. This signal will be denoted by $R_{k}$ and details on this computation can be found in [30].

The EDR will be used to decompose the heart rate and estimate the sympathovagal balance using the proposed approach. This will be done in order to assess whether the EDR poses any limitation in the construction of the respiratory subspace $\mathbb{V}$. Consequently, the applicability of the proposed approach using only the ECG will be analyzed.

4) Statistical Analysis: Two different statistical tests will be used to evaluate the results in this study. First, a comparison between the parameters during the different phases will be performed using the Friedman test for repeated measures. A multicomparison test will be made between the 6 different stress phases with Bonferroni correction equal to $\alpha / 15=$ 0.003 and $\alpha=0.05$. A similar comparison will be performed on the 4 different blockade phases. In addition, a post hoc power analysis will be used to determine the level of statistical power (i.e. Power $=1-\beta$ ) achieved for the sample size used in the study. This analysis will be based on a one-tailed nonparametric test and $\alpha=0.05$, and the effect size will be computed using minimum dispersion in the means and the asymptotic relative efficiency factor.

The second comparison will be performed between the parameters obtained using the real respiratory signals and the EDR. At this point, the Kruskal-Wallis test will be used with $\alpha=0.05$.

\section{RESULTS}

\section{A. Simulation studies}

The MAPE and the percentage errors of the LF, HF, and LFn, calculated using (5), and (6), for both simulations are indicated in Figure 3. The results for the simulation using monocomponent respiratory frequencies (a to d) are indicated as the median, 25th, and 75th percentiles, while for the respiratory signals of the Fantasia dataset (e), the results are indicated for all $1000 \mathbf{Y}_{\text {ANs }}$ signals together. At this point, the goal was to recover the ANS signal after removing all liner interactions of respiration, hence, the error between the recovered signal, namely, $\mathbf{Y}_{\perp}$, and $\mathbf{Y}_{\mathrm{ANS}}$ should be minimal.

1) Monocomponent Respiratory Frequency: The subplots (a) to (d) of Figure 3 depict the median and the 25 th and 75th percentiles of the different performance measures for each frequency. Note that the MAPE (a) and the percent error of the normalized LF component $\left((\mathrm{d}): e_{n}\right)$ are lower than $3 \%$, while the percentage errors in the LF (b) and HF (c) bands are lower than $5 \%$ for all frequencies. These low errors suggest that the heart rate decomposition is able to separate respiratory influences when these overlap with either frequency band, LF or HF. In other words, there is no frequency dependency in the decomposition. For these simulations, the delays computed as the minimum between AIC and MDL ranged from $0.5 \mathrm{~s}$ to $3.6 \mathrm{~s}$, with a median value of $1 \mathrm{~s}$.

2) Broadband Respiration: The occupied bandwidth of the real respiratory signals in this simulation study range between $0.2 \mathrm{~Hz}$ and $0.55 \mathrm{~Hz}$ with mean value of $0.43 \mathrm{~Hz}$ and standard deviation of $0.03 \mathrm{~Hz}$. These values correspond to the range of frequencies where the integrated power is $0.5 \%$ and $99.5 \%$ of the total power. In contrast, for the first simulation, the occupied bandwidth was $0.04 \mathrm{~Hz}$.

The same $1000 \mathbf{Y}_{\text {ANs }}$ signals used in the previous simulation were influenced by the respiratory signals of the Fantasia dataset. The different percent errors are depicted in subplot (e) of Figure 3, and they are indicated for all the respiratory signals together. Note that the MAPE and the error of the normalized LF $\left(e_{n}\right)$ are again the lowest, while the errors in the absolute values of the LF and HF are higher in percentage. At this point, it is clear that the normalized value of LF is kept with a median error of $1.4 \%$ with respect to the LFn of $\mathbf{Y}_{\text {Ans }}$. The delays used for the decomposition ranged from $0.6 \mathrm{~s}$ to $5.3 \mathrm{~s}$, with a median value of $1.4 \mathrm{~s}$.

From these simulations, it is noticeable that the heart rate decomposition is not affected by either the respiratory frequency or the spectral properties (e.g. broadband spectrum), and it is able to reconstruct the original $\mathbf{Y}_{\text {ANs }}$ signal with a median error of $0.7 \%$. In addition, the normalized power of the LF band is retained with an error lower than $2 \%$.

\section{B. Pharmacological autonomic blockade dataset}

Time domain parameters for each protocol and each phase were calculated and they are shown in Figure 4. The root mean square of successive differences between normal heartbeats (RMSSD) is indicated in the top-middle panel. It is clear that during the atropine protocol, these values are significantly lower than during control, which result from an attenuated 


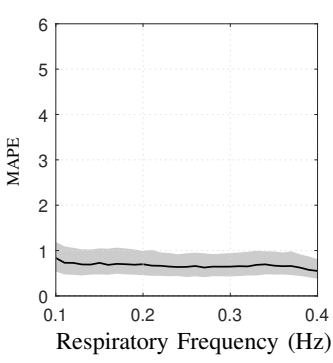

(a)

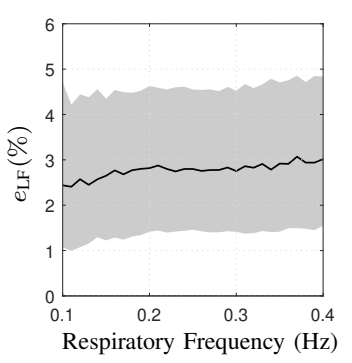

(b)

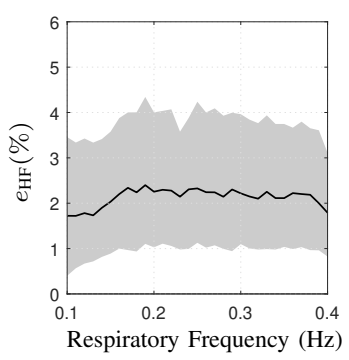

(c)

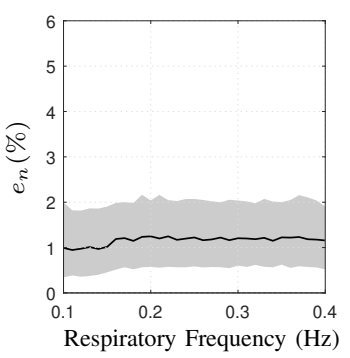

(d)

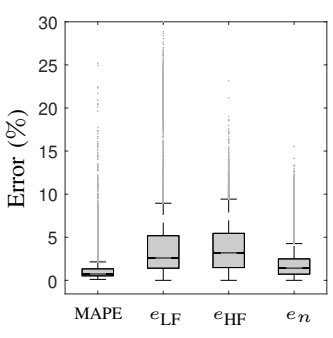

(e)

Fig. 3. Performance measures obtained for the simulation studies. The results of the simulation using the single respiratory frequencies are depicted in the

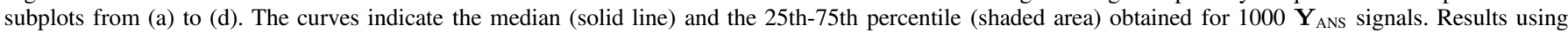
the broadband respiratory signals are indicated in (e) for all signals together. The mean absolute percentage error (MAPE) is computed between $\mathbf{Y}_{\perp}$ and $\mathbf{Y}_{\mathrm{ANS}}$, and the percentage errors $e_{\mathrm{LF}}, e_{\mathrm{HF}}$, and $e_{n}$ are computed between the same signals in the LF, HF, and normalized LF bands.
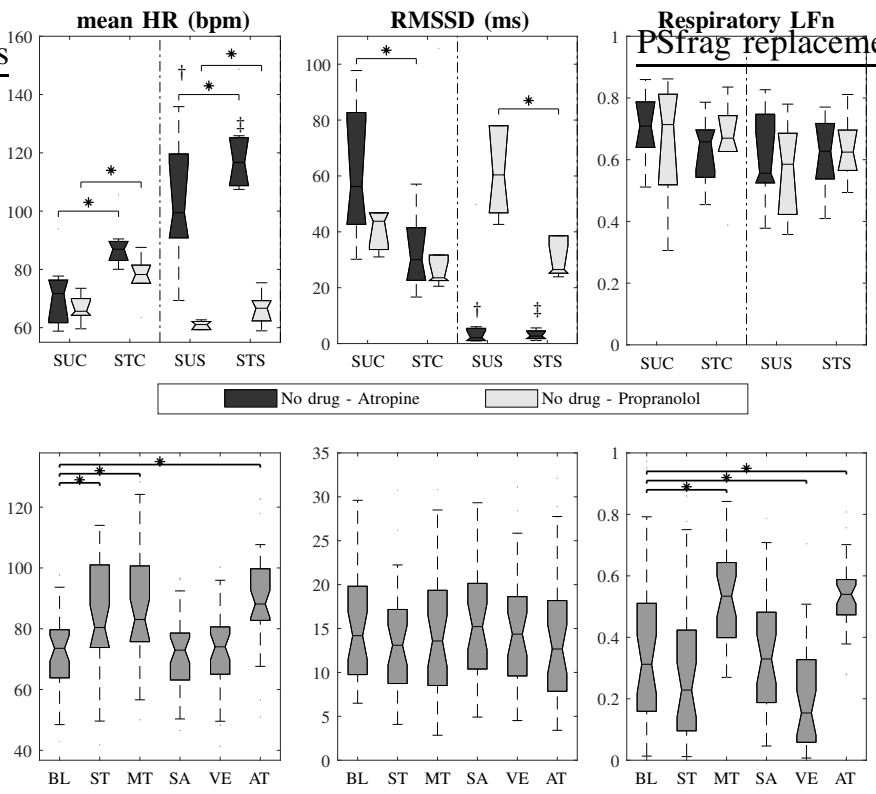

Fig. 4. Mean heart rates, RMSSD, and normalized power in the LF band of the respiratory signal for the pharmacological autonomic blockade dataset (top) and the stress dataset (bottom). SUS and STS refer to single blockade. The $*$ indicates a significant difference of $p$-value $<0.05$ for the blockade dataset and of $p$-value $<0.003$ for the stress dataset. $\dagger$ and $\ddagger$ indicate a significant difference with respect to SUC and STC, respectively.

parasympathetic activity. This can also be observed from the increased mean HR and from the quantification of vagal activity using the traditional $\mathrm{HF}$ power and the total power of the respiratory component $\mathbf{Y}_{\mathbf{X}}$ (see Figure 5). Moreover, the HF tends to underestimate the vagal activity in almost all phases. This can be explained by the fact that more than $50 \%$ of the power of the respiratory signal is contained in the LF band (see Figure 4 top-right), which is caused by the randomized breathing protocol described in Section II-D. This overlap between respiration and the LF band is considered by the Power $\left(\mathbf{Y}_{\mathbf{X}}\right)$, which allows to account for all the linear influences of respiration, including those in the LF band. The underestimation of the vagal activity obtained using the classical HRV analysis is clearly affecting the quantification of the sympathovagal balance, which tends to be higher for $\mathrm{SB}$ than for $\mathrm{SB}_{\mathrm{u}}$ during $\mathrm{SUC}$ for both protocols (see Figure 5). In these two control supine phases, $\mathrm{SB}_{\mathrm{u}}$ is significantly
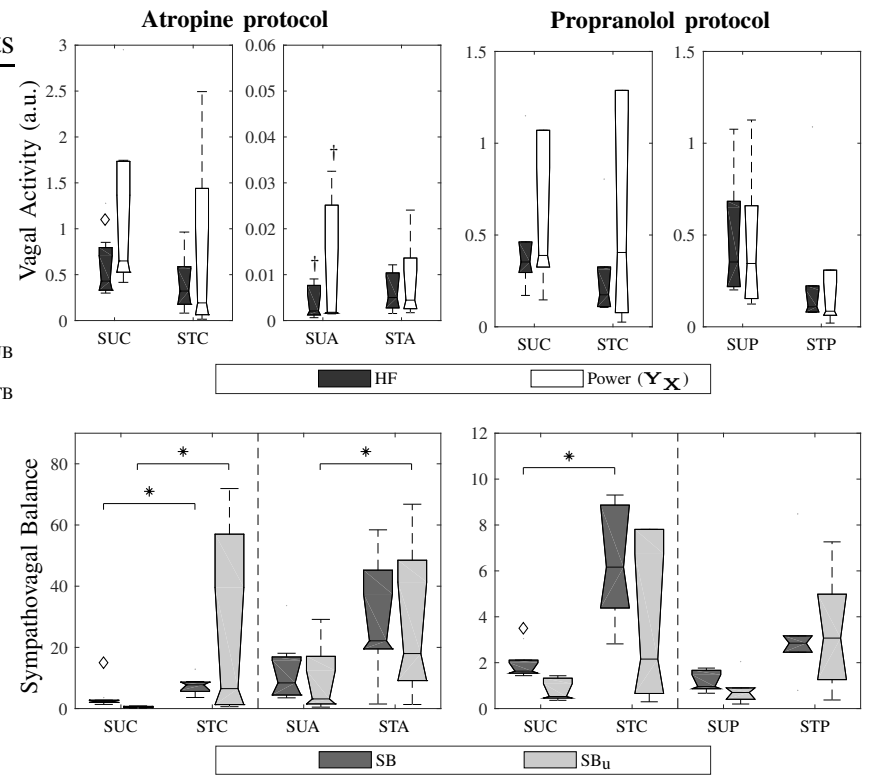

Fig. 5. (top) Vagal activity quantified using the traditional $\mathrm{HF}$ and the the Power $\left(\mathbf{Y}_{\mathbf{X}}\right)$ for the different phases in the pharmacological autonomic blockade dataset. (bottom) Sympathovagal balance calculated using the traditional $\mathrm{SB}=\mathrm{LF} / \mathrm{HF}$ and the proposed index $\mathrm{SBu}$. The * indicates a significant difference of $p$-value $<0.05$ between the phases, the diamond indicates a significant difference with respect to $\mathrm{SBu}_{\mathrm{u}}$, and $\dagger$ indicates a significant difference with respect to SUC.

lower than $\mathrm{SB}$, with a statistical power equal to $1-\beta=0.99$. As a result, a higher estimate of the sympathovagal balance can be achieved by the classical approach only because of an apparent lower vagal activation and not because of an increase in sympathetic activation (see Figure 5, bottom-right). This suggest that an improved quantification of the sympathovagal balance can be obtained by means of $\mathrm{SB}_{\mathrm{u}}$ after removing the respiratory influences in the LF.

When looking at the differences between positions for the parasympathetic (atropine) and sympathetic (propranolol) blockade, the sympathovagal balance always tends to increase when going from supine to standing. This reflects the sympathetic activation expected during this orthostatic challenge, which is captured by both indices, namely, the classical SB and $\mathrm{SB}_{\mathrm{u}}$ for all phases. During SUA and STA, pure sympathetic activation is expected and although a tendency to estimate higher sympathetic activity is observed in both indices, when 
going to STA, a significant difference is only detected by $\mathrm{SB}_{\mathrm{u}}$. During pure parasympathetic activity (i.e. SUP), the tendency of the classical SB of estimating higher sympathetic activity when compared to $\mathrm{SB}_{\mathrm{u}}$ could be also caused by the respiratory influences in LF. However, no significant differences were observed between both indices during this phase.

The model order used for the decomposition of this dataset varied between 1 and $15 \mathrm{~s}$, with a mean value of $2.1 \mathrm{~s}$. The wide variation of model orders was due to the fact that some subjects followed more closely than others the irregular breathing protocol, hence producing a nearly flat respiratory spectrum. As a result, a larger model order was needed to characterize better the respiratory subspace.

\section{Stress Data}

The mean heart rate and the RMSSD were calculated from the tachogram of each subject during each stress phase, and the values are shown in Figure 4 (bottom). It can be seen that no differentiation between BL and stress phases can be done by the RMSSD, despite the increased vagal activity expected during BL and better captured by $\mathcal{P}_{\mathbf{X}}$ (see Figure 6 ). Figure 4 (bottom) also shows the normalized power of the respiratory signal in the LF band, calculated as $\mathrm{LF} /(\mathrm{LF}+\mathrm{HF})$, which is significantly larger during MT and AT, phases where the subjects were asked to speak. During these phases, the mean heart rates are also significantly larger as well as during the story telling (ST) phase, which could be associated with an increase in the stress level. It is clear that during MT and AT the heart rate is higher and the respiratory signal contains information in the LF band, which might interfere with the quantification of the sympathetic activity. This on its turn could potentially reduce the discriminatory power of the classical HRV indices for stress detection, as will be shown later. Furthermore, any methodology to estimate the RSA, based on the frequency content of the respiration, will be affected by sympathetic influences.

After decomposing the HRV signals, the relative powers defined in (3) were compared. Figure 6 shows the results for the relative power of the respiratory component, namely, $\mathcal{P}_{\mathbf{X}}$. Note that all stress phases are significantly lower than baseline (BL), as a vagal withdrawal is expected during stress, which results in a lower RSA. Hence, it is possible to use the $\mathcal{P}_{\mathbf{X}}$ as a way to quantify cardiorespiratory interactions when subjects are breathing naturally and even speaking (i.e. during MT and AT). During these tasks no current RSA algorithm based on the inspiration:expiration ratio nor on the respiratory frequency could be used since the respiratory signal is highly nonstationary. The figure also indicates the normalized LF values for each heart rate component and for the original heart rate variability signal. No differences can be found when performing the classical HRV analysis. This is also the case when looking at $\mathrm{LFn}_{\perp}$, however, it appears that for two stress phases, namely, ST and VE, LFn ${ }_{\mathbf{X}}$ is significantly lower than during BL. These results suggest that during BL, the higher LFn content is mainly the result of the overlap between the respiratory content and the LF band of HRV, as can be observed in Figure 4. The model order for the heart rate decomposition ranged between 1 and $3 \mathrm{~s}$ with a mean of $1.7 \mathrm{~s}$.

Table I shows all the frequency parameters for the original HRV signal $\mathbf{Y}$, the respiratory component $\mathbf{Y}_{\mathbf{X}}$, and the residual component $\mathbf{Y}_{\perp}$. The total power of $\mathbf{Y}_{\mathbf{X}}$, which could be used to quantify RSA, is also indicated for each stress phase. These values are significantly reduced during stress as shown in Table I and Figure 7. The sympathovagal balance values calculated using the classical $(\mathrm{SB})$ and the proposed approach $\left(\mathrm{SB}_{\mathrm{u}}\right)$ are also indicated. Note that the latter and the power of $\mathbf{Y}_{\mathbf{X}}$ allow to differentiate between stress phases and baseline, since the respiratory influences are removed from the LF band of HRV. This is clear from Figure 6, where both $\mathrm{SB}$ and $\mathrm{SB}_{\mathrm{u}}$ are indicated. None of the phases is different from baseline when using the traditional SB, while the unconstrained approach clearly shows differences when an increased sympathetic activity is expected.

The statistical power of the different evaluations was estimated and for a sample size of 35, an effect size of 0.5 and $\alpha=0.05$, the statistical power was equal to $1-\beta=0.91$. This means that there was a $9 \%$ chance of making a type II error. In other words there was only a $9 \%$ of chance of obtaining the same parameter values for BL and all stress phases.

In order to determine whether the proposed estimation of the RSA and the sympathovagal balance could also be derived using the ECG-derived respiration (EDR), the algorithm based on kPCA was used to extract the EDR that was then used to decompose the heart rate. The results obtained for the EDR signal on the stress dataset are depicted in Figure 7 together with those obtained with the real respiration. No significant differences were found between the values obtained using the EDR and the real respiration. Furthermore, all differences between BL and all the stress phases were reproduced by the EDR, which indicates that under these conditions the respiratory subspace $\mathbb{V}$ can also be constructed using this derived signal. Other algorithms for EDR calculation, such as the R-peak amplitude [28], PCA [29], and slopes and angles of the QRS [31], were also tested. The best results, in terms of differences between phases and resemblance with the real respiration, were obtained with the kPCA algorithm. These results are not presented here since an EDR comparison is out of the scope of this work.

\section{Discussion}

This study proposed a new methodology to better estimate the RSA and the sympathovagal balance after separating respiratory influences from the HRV. This was achieved after decomposing the heart rate into two components. One of these components describes the linear influences of respiration in heart rate and this was proven using three different experiments. First, the simulation study showed that the decomposition can indeed remove all linear respiratory information from the heart rate while keeping the residual dynamics even when they are contained in the same frequency band as the respiratory signal. This is important since it allows to quantify the LF component of HRV without the influence of respiration. This was tested for different frequencies and 

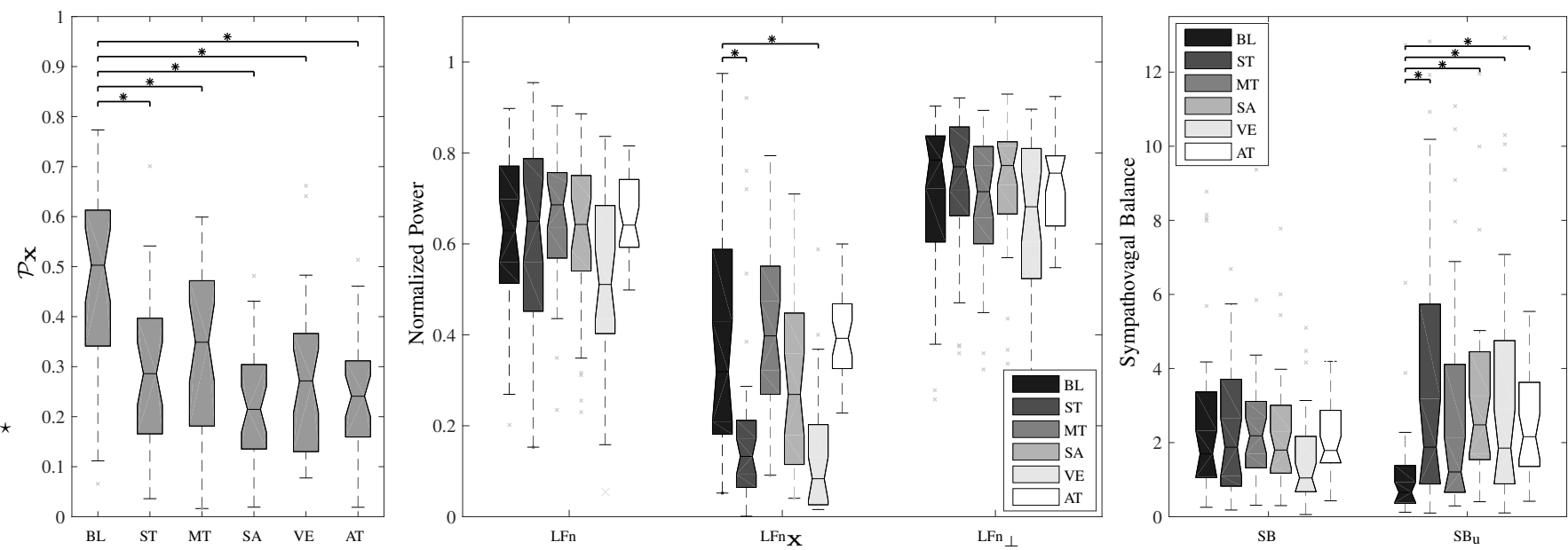

Fig. 6. Relative power of the respiratory component $\left(\mathcal{P}_{\mathbf{X}}\right)$, normalized powers of the original HRV signal $(\mathrm{LFn})$, the respiratory component $\left(\mathrm{LFn} \mathrm{X}_{\mathbf{X}}\right)$, and the residual component $\left(\mathrm{LFn}_{\perp}\right)$, and sympathovagal balance computed using the classical approach $(\mathrm{SB})$ and the proposed methodology $(\mathrm{SB} \mathrm{u})$ for each stress phase. The * indicates a significant difference of $p$-value $<0.003$.

TABLE I

MEDIAN \pm MAD OF THE FREQUENCY PARAMETERS FOR EACH STRESS PHASE, AND DERIVED FROM EACH HEART RATE COMPONENT, NAMELY, THE RESPIRATORY COMPONENT $\mathbf{Y}_{\mathbf{X}}$ AND THE RESIDUAL COMPONENT $\mathbf{Y}_{\perp}$.

\begin{tabular}{|c|c|c|c|c|c|c|c|}
\hline Component & HRV index & BL & ST & MT & SA & VE & AT \\
\hline $\mathbf{Y}$ & $\begin{array}{l}\text { LF } \\
\text { HF } \\
\text { LFn } \\
\text { SB }\end{array}$ & $\begin{array}{c}142.48 \pm 39.10 \\
90.88 \pm 39.99 \\
0.62 \pm 0.16 \\
1.69 \pm 2\end{array}$ & $\begin{array}{c}106.15 \pm 49.88 \\
67.12 \pm 38.33 \\
0.64 \pm 0.18 \\
1.87 \pm 2.13\end{array}$ & $\begin{array}{c}159.10 \pm 41.21 \\
76.31 \pm 25.32 \\
0.68 \pm 0.12 \\
2.18 \pm 1.25\end{array}$ & $\begin{array}{c}147.46 \pm 34.28 \\
76.24 \pm 31.46 \\
0.64 \pm 0.13 \\
1.79 \pm 1.24\end{array}$ & $\begin{array}{c}115.15 \pm 34.52^{*} \\
112.88 \pm 37.89 \\
0.51 \pm 0.15 \\
1.04 \pm 0.96\end{array}$ & $\begin{array}{c}143.09 \pm 31.52 \\
80.43 \pm 25.99 \\
0.64 \pm 0.10 \\
1.78 \pm 1.12\end{array}$ \\
\hline $\mathbf{Y}_{\mathbf{X}}$ & $\begin{array}{c}\mathrm{LF}_{\mathbf{X}} \\
\mathrm{HF}_{\mathbf{X}} \\
\mathrm{LFn}_{\mathbf{X}} \\
\mathrm{SB}_{\mathbf{X}} \\
\text { Total Power }\end{array}$ & $\begin{array}{c}33.26 \pm 37.71 \\
62.14 \pm 34.14 \\
0.31 \pm 0.23 \\
0.46 \pm 4.07 \\
\mathbf{1 1 7 . 3 5} \pm \mathbf{4 7 . 7 2}\end{array}$ & $\begin{array}{c}4.98 \pm 9.42^{*} \\
36.82 \pm 31.16 \\
0.13 \pm 0.14^{*} \\
0.15 \pm 0.93^{*} \\
\mathbf{5 7 . 3 3} \pm \mathbf{4 0 . 8 6}^{*}\end{array}$ & $\begin{array}{c}26.88 \pm 19.89 \\
41.94 \pm 18.85 \\
0.39 \pm 0.13 \\
0.66 \pm 0.55 \\
\mathbf{7 5 . 3 5} \pm \mathbf{4 0 . 5 5}\end{array}$ & $\begin{array}{c}9.73 \pm 10.03^{*} \\
34.05 \pm 17.66^{*} \\
0.26 \pm 0.16 \\
0.36 \pm 0.40 \\
\mathbf{5 1 . 7 5}^{*} \mathbf{2 8 . 2 1}{ }^{*}\end{array}$ & $\begin{array}{c}4.58 \pm 6.09^{*} \\
53.60 \pm 27.14 \\
0.08 \pm 0.10^{*} \\
0.09 \pm 0.18^{*} \\
\mathbf{5 6 . 9 6} \pm \mathbf{3 6 . 9 8}^{*}\end{array}$ & $\begin{array}{c}20.36 \pm 11.42 \\
31.13 \pm 14.85^{*} \\
0.39 \pm 0.09 \\
0.64 \pm 0.34 \\
\mathbf{5 5 . 5 7}^{*} \mathbf{3 0 . 8 0}^{*}\end{array}$ \\
\hline $\mathbf{Y}_{\perp}$ & $\begin{array}{c}\mathrm{LF}_{\perp} \\
\mathrm{HF}_{\perp} \\
\mathrm{LFn}_{\perp} \\
\mathrm{SB}_{\perp}\end{array}$ & $\begin{array}{c}82.64 \pm 34.02 \\
23.44 \pm 14.48 \\
0.78 \pm 0.13 \\
3.63 \pm 1.78\end{array}$ & $\begin{array}{c}98.52 \pm 49.39 \\
30.59 \pm 13.67 \\
0.76 \pm 0.13 \\
3.34 \pm 2.46\end{array}$ & $\begin{array}{c}83.17 \pm 48.18 \\
39.92 \pm 16.01^{*} \\
0.71 \pm 0.12 \\
2.50 \pm 1.81\end{array}$ & $\begin{array}{c}122.82 \pm 35.22^{*} \\
39.11 \pm 19.06^{*} \\
0.77 \pm 0.12 \\
3.39 \pm 1.84\end{array}$ & $\begin{array}{c}101.60 \pm 39.03 \\
47.10 \pm 21.25^{*} \\
0.68 \pm 0.15 \\
2.14 \pm 1.93\end{array}$ & $\begin{array}{c}115.65 \pm 33.39 \\
38.90 \pm 14.43^{*} \\
0.75 \pm 0.10 \\
3.09 \pm 1.58\end{array}$ \\
\hline $\mathbf{Y}_{\mathbf{X}}, \mathbf{Y}_{\perp}$ & $\mathbf{S B}_{\mathrm{u}}$ & $0.65 \pm 1.24$ & $1.87 \pm 4.13^{*}$ & $1.20 \pm 4.7$ & $2.47 \pm 5.51^{*}$ & $1.85 \pm 2.67^{*}$ & $2.15 \pm 4.33^{*}$ \\
\hline
\end{tabular}

* Significant difference with respect to baseline (BL) with a $p$-value $<0.003$.

different characteristics of the respiratory signal and the errors between the simulated ANS signal and the residual heart rate $\mathbf{Y}_{\perp}$ were on average lower than $1 \%$ and the $\mathrm{LF}$ and $\mathrm{HF}$ powers could be recovered with an error lower than $5 \%$. These results indicate that the heart rate decomposition does not have any frequency dependence. In other words, the decomposition works when the respiration has a single fundamental frequency (i.e. peaked spectrum) and when it has a broadband spectrum. At this point it is clear that when performing HRV analysis, no constraints on the spectrum of the respiration need to be imposed. Furthermore, the fact that the decomposition works for broadband respiratory spectra is an important advantage of the proposed methodology for real-life applications, such as during spontaneous breathing or even speaking.

The second study was performed on a pharmacological protocol and the results showed that the proposed indices can quantify both the RSA and the sympathovagal balance under sympathetic and parasympathetic withdrawal. These results were compared against the classical HRV indices (e.g. RMSSD and LFn) and it was demonstrated that the proposed approach corrects for respiratory influences in the LF band and it allows to identify increases in sympathetic activity. This was also tested while subjects changed from supine to standing positions. At this point, the proposed indices can capture changes in the sympathetic activity due to an orthostatic challenge. This was demonstrated since $\mathrm{SB}_{\mathrm{u}}$ identified increases in sympathetic activity during parasympathetic blockade while the classical SB tend to fail. As a result, the proposed quantification of the sympathovagal balance is less affected by reductions in the denominator (i.e. vagal activity), which can lead to apparent increases in sympathetic activity when using the classical HRV.

The third study was performed on data collected during a stress test and the results showed again that the sympathetic activations during stress can be identified even when they are masked by the respiratory dynamics. The overlap between 
respiration and heart rate in the LF band was confirmed for both datasets and it was shown that in phases where subjects were speaking this overlap was largest. In fact, this is an important disadvantage of state-of-the-art approaches for RSA quantification [8], since at this point, neither an inspiratory:expiratory ratio nor a clear respiratory frequency could be defined [3]. In contrast, this study demonstrated that this overlap could be tackled using the proposed approach. Figures 5 and 6 and Table I showed that during control phases in the pharmacological dataset and during BL in the stress dataset, a higher content of respiratory information is present in the heart rate. This is observed in the higher power of the respiratory component during these phases, which could be associated with a stronger cardiorespiratory coupling, hence, a larger RSA. If the classical HRV analysis is performed in the stress set, it is clear that no difference can be observed between any of the stress phases and BL, except for the LF power during VE. This is again a result of the aforementioned overlap. Instead, after removing all respiratory influences from the heart rate, the sympathovagal balance during BL is significantly lower than during ST, SA, VE, and AT. These findings confirm the results reported in [3], where a difference in the LFn was found between BL and ST and SA after correcting the HF band and after removing segments where the respiratory rate overlapped with the LF band. The HF band correction was applied in order to better quantify the vagal modulation of HRV, and the segments where respiratory rate overlapped with the LF band were removed from the analysis in order not to mix sympathetic and parasympathetic activities. Apart from these corrections, a constraint of peaked respiratory spectra was imposed in [3], which resulted in the removal of additional segments. In this study, these constraints and adaptation were no longer needed, hence, all segments were included in the analysis and all results were still consistent with those reported in [3]. Therefore, the expected vagal withdrawal and the increased sympathetic activation during stress [1] were quantified by the reduced linear respiratory influences $\left(\mathrm{LF}_{\mathbf{X}}\right.$, and $\left.\mathrm{HF}_{\mathbf{X}}\right)$, the estimated RSA (i.e. the total power of $\mathbf{Y}_{\mathbf{X}}$ ), and the improved calculation of the sympathovagal balance. All these results are in agreement with [3] in that considering respiratory information for ANS analysis during stress is highly relevant. These results were also confirmed by the pharmacological data, where autonomic blockade was imposed and the proposed indices could indeed capture changes of the sympathetic activation unrelated to parasympathetic activity during the blockade and during an orthostatic challenge.

The improved quantification of the RSA can be clearly observed during BL in the stress dataset (see Figures 4 and 6). Traditional parameters such as the RMSSD and HF cannot capture differences between this phase and the stress phases, while $\mathcal{P}_{\mathbf{X}}$ can. During BL, subjects were listening to a guided relaxation audio, which could be compared to sessions of mindfulness, biofeedback or meditation exercises. Therefore, an increased vagal activation was expected but it was only quantified by the proposed indices. These results suggest that $\mathcal{P}_{\mathbf{X}}$ could potentially be used in biofeedback exercises since it estimates vagal activity better than the classical parameters.

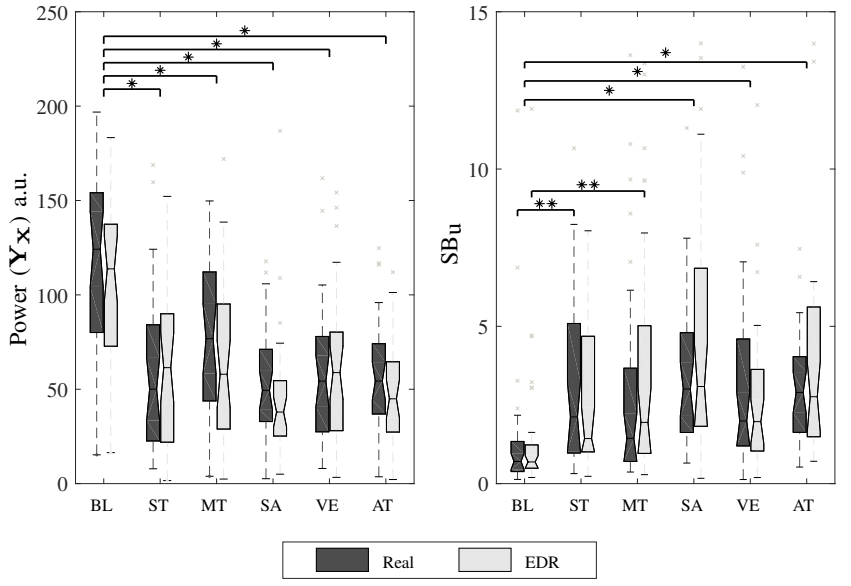

Fig. 7. Proposed index for the RSA estimation (left) and $\mathrm{SB}_{\mathrm{u}}$ calculated using the proposed approach and the EDR signal. No significant differences were found between the EDR and the real respiration. A significant difference between BL and any stress phase for both signals is indicated by *, while ** indicates a significant difference obtained by either of the signals.

As mentioned before, an advantage of the proposed approach is that it can be computed even when the subjects are speaking (e.g. during MT and AT). At this point, it is important to remember that speaking produces changes in heart rate and blood pressure that seem to be unrelated to the stress task, in particular to mental stress [8]. Furthermore, speaking interferes with the natural breathing since a reduced airflow and changes in the lung volume have been observed during natural breathing [32] and in high respiratory drive conditions [33]. This interference can be seen in the respiratory effort, which on its turn will affect the construction of the respiratory subspace. Since the proposed approach considers solely the linear interactions between respiration and heart rate, it is possible to think that during speaking, not all effects of respiration are taken into account by the heart rate decomposition. Even though the broadband spectrum can be modeled by this approach, it is important to keep in mind that $\mathbf{Y}_{\perp}$ does not only describe mechanisms different from respiration, but also the possible nonlinear cardiorespiratory interactions. This could explain the improved sympathovagal results obtained during MT. Whereas these interactions play an important role in the cardiac control, this work only focuses on the linear interactions between respiration and heart rate. Another important point to consider is that the current approach does not consider time-variant properties typical in long-term recordings. To tackle this, other algorithms such as Kalman filter or adaptive filters (e.g. least mean squares (LMS), recursive least squares) could be used. In fact, a classical LMS was tested and after parameter tuning, similar results were obtained. In other words, LMS models with different step sizes were capable to identify differences between BL and ST, MT, VE, and AT, as the OSP model used in this study does. However, for the LMS, the step size has a strong influence in the estimation of the proposed index and even though different techniques to better assess this parameter could be used, the selected methodology is enough for this estimation due to its simplicity and the lack of tuning parameters [4]. Even though 
adaptive algorithms require parameter tuning and are often associated with high computational costs, they might lead to similar or better results in the estimation of the sympathovagal balance for long-term nonstationary signals.

The improved calculation of the RSA and the sympathovagal balance was also evaluated using the EDR signal and all differences between baseline and stress were successfully reproduced. Also, similar parameters were obtained using the EDR in the pharmacological dataset, except during a supine control phase. kPCA takes into account morphology changes of the whole QRS complex, while other state-of-theart algorithms, tested for this purposes, rely on fewer points such as the R-peak, S-wave, Q-wave, or a combination of those. Even though kPCA typically outperforms other EDR techniques, it is known that PCA-based algorithms are the least robust to artefacts [34], which can be seen as a disadvantage when detecting stress in ambulatory settings. In fact, despite obtaining slightly poorer performance than kPCA, the algorithms based on the R-peak amplitude, slopes and angles of the QRS could be more robust in noisy or nonstationary ECG signals [31]. In this study, the results suggest that kPCA could be used to detect the higher sympathovagal balance and lower RSA during stress. However, this is constrained by the fact that the signals in the stress dataset were collected in a controlled environment. In the pharmacological dataset, on the other hand, the breathing protocol was randomized and variable depths and shapes were included in the study. This challenged the construction of the respiratory subspace using the EDR and the algorithm was not able to fully describe respiratory variations in this unnatural and imposed breathing protocol. Therefore, future work will focus on using this novel approach on different long-term datasets with different quality and where a vagal withdrawal and an increased sympathetic activity are expected during natural breathing. Examples of such datasets include sleep apnea events and epileptic seizures.

\section{CONCLUSION}

A methodology to estimate both the RSA and the sympathovagal balance after removing all linear respiratory information from the heart rate was proposed. First, the heart rate was decomposed into a respiratory component and a residual component that describes sympathetic modulations and other, possibly nonlinear, respiratory interactions. This decomposition was performed using orthogonal subspace projections. From the two heart rate components, the powers in the classical LF and in the extended HF bands were computed and then used to estimate the RSA and the sympathovagal balance in an unconstrained way. Results on simulated and real life data proved that, on the one hand, the decomposition is able to keep the LF dynamics of heart rate that are unrelated to respiration. This can be achieved independently of the properties of the respiratory signal, namely the frequency and its spectrum. On the other hand, the estimated RSA and sympathovagal balance identified the vagal withdrawal and sympathetic activation during autonomic blockade and during emotional and mental stress. This is an advantage of the proposed approach over the classical HRV analysis, which has been proven to be inaccurate in situations when the respiratory information falls within the LF band. Another advantage is that this approach allows to quantify the RSA when the respiratory frequency and the inspiratory and expiratory phases cannot be determined.

The estimation of the RSA and the sympathovagal balance was also tested using the EDR, and the results were similar to those obtained with the real respiration. This is important in the development of wearable systems for stress monitoring because this approach can be used to assess both, cardiac and respiratory information using solely the ECG.

\section{REFERENCES}

[1] Task Force ESC and NASPE, "Heart rate variability: standards of measurement, physiological interpretation and clinical use," Circulation, vol. 93, no. 5, pp. 1043-1065, Mar 1996.

[2] G. G. Berntson, J. T. Cacioppo, and K. S. Quigley, "Respiratory sinus arrhythmia: autonomic origins, physiological mechanisms, and psychophysiological implications," Psychophysiology, vol. 30, no. 2, pp. 183-196, 1993.

[3] A. Hernando, J. Lázaro, E. Gil, A. Arza, J. M. Garzón, R. López-Antón, C. de la Cámara, P. Laguna, J. Aguiló, and R. Bailón, "Inclusion of respiratory frequency information in heart rate variability analysis for stress assessment," IEEE journal of biomedical and health informatics, vol. 20, no. 4, pp. 1016-1025, 2016.

[4] D. Widjaja, A. Caicedo, E. Vlemincx, I. Van Diest, and S. Van Huffel, "Separation of respiratory influences from the tachogram: a methodological evaluation," PloS one, vol. 9, no. 7, p. e101713, 2014.

[5] R. Bailón, P. Laguna, L. Mainardi, and L. Sornmo, "Analysis of heart rate variability using time-varying frequency bands based on respiratory frequency," in Engineering in Medicine and Biology Society, 2007. EMBS 2007. 29th Annual International Conference of the IEEE. IEEE, 2007, pp. 6674-6677.

[6] R. Barbieri, J. K. Triedman, and J. P. Saul, "Heart rate control and mechanical cardiopulmonary coupling to assess central volume: a systems analysis," American Journal of Physiology-Regulatory, Integrative and Comparative Physiology, vol. 283, no. 5, pp. R1210-R1220, 2002.

[7] J. W. Denver, S. F. Reed, and S. W. Porges, "Methodological issues in the quantification of respiratory sinus arrhythmia," Biological psychology, vol. 74, no. 2, pp. 286-294, 2007.

[8] R. Sloan, J. Korten, and M. M. Myers, "Components of heart rate reactivity during mental arithmetic with and without speaking," Physiology \& behavior, vol. 50, no. 5, pp. 1039-1045, 1991.

[9] M. Pagani, F. Lombardi, S. Guzzetti, O. Rimoldi, R. Furlan, P. Pizzinelli, G. Sandrone, G. Malfatto, S. Dell'Orto, and E. Piccaluga, "Power spectral analysis of heart rate and arterial pressure variabilities as a marker of sympatho-vagal interaction in man and conscious dog." Circulation research, vol. 59, no. 2, pp. 178-193, 1986.

[10] G. E. Billman, "The lf/hf ratio does not accurately measure cardiac sympatho-vagal balance," Frontiers in physiology, vol. 4, p. 26, 2013.

[11] W. von Rosenberg, T. Chanwimalueang, T. Adjei, U. Jaffer, V. Goverdovsky, and D. P. Mandic, "Resolving ambiguities in the lf/hf ratio: Lf-hf scatter plots for the categorization of mental and physical stress from hrv," Frontiers in physiology, vol. 8, p. 360, 2017.

[12] C. Kouakam, D. Lacroix, N. Zghal, R. Logier, D. Klug, P. Le Franc, M. Jarwe, and S. Kacet, "Inadequate sympathovagal balance in response to orthostatism in patients with unexplained syncope and a positive head up tilt test," Heart, vol. 82, no. 3, pp. 312-318, 1999.

[13] M. Pagani, G. Mazzuero, A. Ferrari, D. Liberati, S. Cerutti, D. Vaitl, L. Tavazzi, and A. Malliani, "Sympathovagal interaction during mental stress. a study using spectral analysis of heart rate variability in healthy control subjects and patients with a prior myocardial infarction," Circulation, vol. 83, no. 4 Suppl, pp. II43-51, 1991.

[14] J. Taelman, S. Vandeput, A. Spaepen, and S. Van Huffel, "Influence of mental stress on heart rate and heart rate variability," in 4th European conference of the international federation for medical and biological engineering. Springer, 2009, pp. 1366-1369.

[15] E. Vanninen, A. Tuunainen, M. Kansanen, M. Uusitupa, and E. Länsimies, "Cardiac sympathovagal balance during sleep apnea episodes," Clinical Physiology and Functional Imaging, vol. 16, no. 3, pp. 209-216, 1996. 
[16] H. Evrengül, H. Tanriverdi, D. Dursunoglu, A. Kaftan, O. Kuru, U. Unlu, and M. Kilic, "Time and frequency domain analyses of heart rate variability in patients with epilepsy," Epilepsy research, vol. 63, no. 2, pp. 131-139, 2005.

[17] J. Choi and R. Gutierrez-Osuna, "Removal of respiratory influences from heart rate variability in stress monitoring," IEEE Sensors Journal, vol. 11, no. 11, pp. 2649-2656, 2011.

[18] C. Varon, A. Caicedo, D. Testelmans, B. Buyse, and S. Van Huffel, "A novel algorithm for the automatic detection of sleep apnea from singlelead ECG," IEEE Transactions on Biomedical Engineering, vol. 62, no. 9, pp. 2269-2278, 2015.

[19] G. Lenis, M. Kircher, J. Lázaro, R. Bailón, E. Gil, and O. Doessel, "Separating the effect of respiration on the heart rate variability using Granger's causality and linear filtering," Biomedical Signal Processing and Control, vol. 31, pp. 272-287, 2017.

[20] L. S. Goldoozian, E. Zahedi, and V. Zarzoso, "Time-varying assessment of heart rate variability parameters using respiratory information," Computers in Biology and Medicine, vol. 89, pp. 355-367, 2017.

[21] S. Wold, H. Antti, F. Lindgren, and J. Öhman, "Orthogonal signal correction of near-infrared spectra," Chemometrics and Intelligent laboratory systems, vol. 44, no. 1-2, pp. 175-185, 1998.

[22] A. Caicedo, C. Varon, B. Hunyadi, M. Papademetriou, I. Tachtsidis, and S. Van Huffel, "Decomposition of near-infrared spectroscopy signals using oblique subspace projections: applications in brain hemodynamic monitoring," Frontiers in physiology, vol. 7, 2016.

[23] J. P. Saul, R. D. Berger, P. Albrecht, S. Stein, M. H. Chen, and R. Cohen, "Transfer function analysis of the circulation: unique insights into cardiovascular regulation," American Journal of Physiology-Heart and Circulatory Physiology, vol. 261, no. 4, pp. H1231-H1245, 1991.

[24] M. Orini, R. Barbieri, M. Nardelli, E. P. Scilingo, and G. Valenza, "Introduction to complex cardiovascular physiology," in Complexity and Nonlinearity in Cardiovascular Signals. Springer, 2017, pp. 3-42.

[25] C. Bainton, D. Richter, H. Seller, D. Ballantyne, and J. Klein, "Respiratory modulation of sympathetic activity," Journal of the autonomic nervous system, vol. 12, no. 1, pp. 77-90, 1985.

[26] N. Iyengar, C. Peng, R. Morin, A. L. Goldberger, and L. A. Lipsitz, "Age-related alterations in the fractal scaling of cardiac interbeat interval dynamics," American Journal of Physiology-Regulatory Integrative and Comparative Physiology, vol. 40, no. 4, p. R1078, 1996.

[27] J. Mateo and P. Laguna, "Analysis of heart rate variability in the presence of ectopic beats using the heart timing signal," IEEE Transactions on Biomedical Engineering, vol. 50, no. 3, pp. 334-343, 2003.

[28] G. B. Moody et al, "Derivation of respiratory signals from multi-lead ECGs," Proc. Computers in cardiology, vol. 12, no. 1985, pp. 113-116, 1985.

[29] P. Langley et al, "Principal component analysis as a tool for analyzing beat-to-beat changes in ECG features: application to ECG-derived respiration," IEEE Transactions on Biomedical Engineering, vol. 57, no. 4, pp. 821-829, Apr 2010.

[30] D. Widjaja et al, "Application of kernel principal component analysis for single-lead-ECG-derived respiration," IEEE Transactions on Biomedical Engineering, vol. 59, no. 4, pp. 1169-1176, Apr 2012.

[31] J. Lázaro, A. Alcaine, D. Romero, E. Gil, P. Laguna, E. Pueyo, and R. Bailón, "Electrocardiogram derived respiratory rate from QRS slopes and R-wave angle," Annals of biomedical engineering, vol. 42, no. 10, pp. 2072-2083, 2014.

[32] J. D. Hoit, B. L. Plassman, R. W. Lansing, and T. Hixon, "Abdominal muscle activity during speech production," Journal of Applied Physiology, vol. 65, no. 6, pp. 2656-2664, 1988.

[33] E. F. Bailey and J. D. Hoit, "Speaking and breathing in high respiratory drive," Journal of Speech, Language, and Hearing Research, vol. 45, no. 1, pp. 89-99, 2002.

[34] C. Varon and S. Van Huffel, "ECG-derived respiration for ambulatory monitoring," in Computing in Cardiology Conference (CinC), 2015. IEEE, 2015, pp. 169-172. 